\title{
Urea cycle disorder drug approved
}

Hyperion Therapeutics' first drug, approved in February by the US Food and Drug Administration, has been welcomed by clinicians as a step forward for people with rare genetic conditions known as urea cycle disorders (UCDs). Ravicti (glycerol phenylbutyrate), an orphan drug from S. San Francisco, Californiabased Hyperion, got the agency's nod based on a 44-patient phase 3 trial showing that it was as effective in controlling blood ammonia levels as the standard-of-care Buphenyl (sodium phenylbutyrate) from Valeant Pharmaceuticals, of Montreal.

Individuals with UCDs have deficiencies in enzymes involved in the urea cycle, the body's main mechanism for removing ammonia from blood. Ammonia is a potent neurotoxin, resulting from the natural breakdown of protein and nucleic acids. Normally, in a series of enzymatic steps, the urea cycle transforms this neurotoxic nitrogen waste into urea, which is excreted as urine by the kidneys. In people with UCDs, if left untreated, ammonia levels will increase, resulting in brain damage and death. Hyperion estimates the prevalence of UCD deficiencies in the US is about 2,000.

Both Buphenyl and Ravicti provide an alternative sink to the urea cycle, acting as nitrogen scavenger drugs. The drugs are both metabolized to the same active substance, phenylacetic acid (PAA). This metabolite is conjugated with the amino acid glutamine (a temporary storage form of ammonia) to form phenylacetylglutamine, which is then eliminated by the kidneys. Buphenyl is a pro-drug of PAA, whereas Ravicti is a pre-pro-drug of PAA.

Although the two drugs operate through a similar mechanism, Ravicti represents an "evolutionary step forward in the management of UCDs," says Marshall Summar, director of clini-

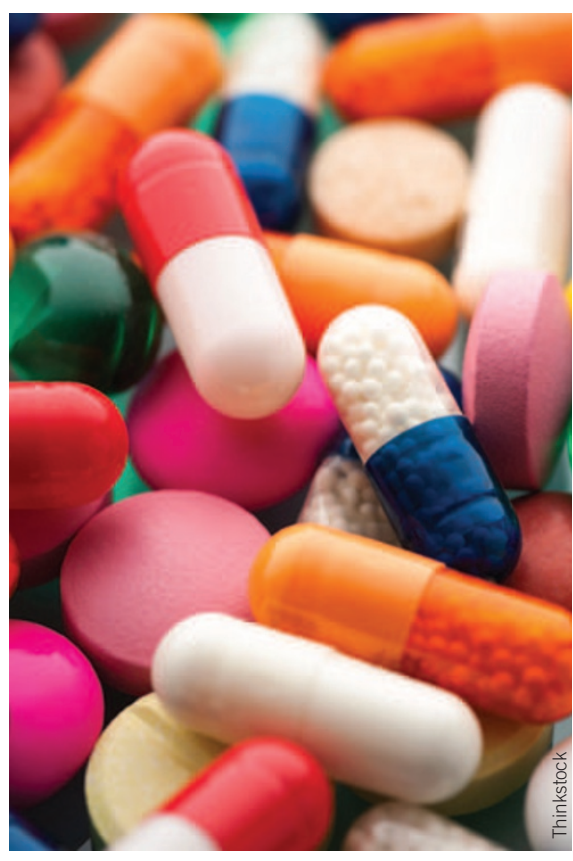

Up to 40 pills a day might be taken by individuals with urea cycle disorder to keep blood ammonia levels under control.

cal research at the Children's National Medical Center (CNMC) in Washington, DC, because of side effects associated with Buphenyl. The latter drug is not well tolerated, mainly due to its unpleasant taste and gastrointestinal side effects, high sodium content and high pill burden (up to 40 pills, administered three to six times a day). In contrast, Ravicti is almost tasteless and is formulated as a concentrated liquid: about one teaspoon three times a day is equivalent to 40 tablets of Buphenyl. Ravicti also helps prevent some of the gastrointestinal side effects associated with Buphenyl as it is a slow-release formu- lation broken down by pancreatic enzymes in the intestine rather than in the stomach.

All this means that clinicians expect better compliance with Ravicti than Buphenyl, and this may be crucial, says CNMC executive vice president and chief academic officer Mark Batshaw, especially for young children in whom UCDs are often diagnosed. "Missing doses can be life threatening, so adherence is important in terms of outcome," agrees Brendan Lee of Baylor College of Medicine in Houston, who served as the lead investigator of Ravicti's phase 3 trial.

If cost were not a consideration, most physicians say they would give Ravicti to all eligible patients. But Ravicti is priced at $\$ 250,000$ $\$ 290,000$ /year, much higher than Buphenyl, which analysts at Needham in New York estimate as $\$ 40,000-50,000 /$ year for children under $20 \mathrm{~kg}$ and $\$ 100,000-130,000 /$ year for adults (prices are difficult to calculate because dosing is based on weight). Hyperion has until the end of April to decide whether to exercise an option to purchase Buphenyl from Valeant, which analysts expect it to do. Buphenyl is also now an off-patent drug opening opportunities for generics. For now, though, most doctors are likely to continue to start affected infants on Buphenyl (so far, Ravicti has not been approved for children under 2).

Payors are likely to reimburse Ravicti for those who opt for it over Buphenyl, says analyst Joseph Schwartz at Leerink Swann in Boston. Needham analyst Alan Carr agrees, citing the tolerability advantage and "relatively limited cost to payors overall," given the small patient population. Annual peak sales of Ravicti in UCD are predicted to be at least $\$ 100$ million. Hyperion is also planning a phase 3 trial of Ravicti in hepatic encephalopathy, a condition where ammonia is also a major toxin.

Malini Guha Freehold, New Jersey

\section{IN their words}

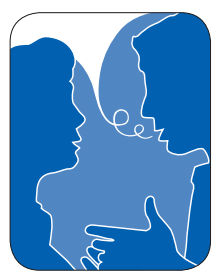

"We love the researchers but they don't know the social aspects of this," says Stuart Siedman, father of a child with a rare genetic disorder. Parents like Siedman are trying to get researchers to share clinical trial data. Drug developers say it's not that easy. (The Wall Street Journal, 18 February 2013)

"This is such a win-win for both medicine and basic science, demonstrating what we can achieve when we work together," Lawrence Bonassar, biomedical engineer at Weill Cornell Medical College in Ithaca,
New York, describing the project that produced an artificial ear by three-dimensional printing. (Mail Online, 21 February 2013)

"We realized we both care about the same things, so why should we have Sage over here doing one thing, and Dream over there doing another?" Stephen Friend, co-founder and CEO of SAGE, says of its merger with the all-volunteer DREAM (dialogue on reverse engineering assessment and methods) project. Both groups espouse open access and collaboration. (Xconomy, 19 February 2013)

"In this case, we have a more difficult and fascinating question of what are brainwide activity patterns and ultimately how do they make things happen?" Ralph Greenspan, of the Kavli Institute for Brain and Mind at the University of California, San Diego, comparing the newly announced Brain
Activity Map Project to the Human Genome Project. (The New York Times, 17 February 2013)

"I may be a bit of a cheerleader and a catalyst, but the students have been fantastic." Bob Langer of MIT muses on his career upon receiving the Medal of Technology and Innovation from the White House. Langer is one of only seven people to win both this and the Medal of Science, which was awarded to him in 2007. (WGBH News, 22 February 2013)

"[Such pricing] sends the message that all this investment in research and all this talk about innovation is, at the end of the day, not going to be rewarded." John Lechleiter, CEO of Eli Lilly of Indianapolis, wondering what price controls in Europe portends for drug development. (The Wall Street Journal, 6 March 2013) 